\title{
Smart Streaming of Panoramic Videos
}

\author{
Hongwei Ma \\ The Chinese University of Hong Kong \\ Zhiyou Ma \\ Kandao Technology
}

\author{
Xiaoke Jiang \\ Kandao Technology \\ Yizhen Cai \\ The Chinese University of Hong Kong
}

\begin{abstract}
The streaming of panoramic videos comes with a new challenge: adapting the streamed content to user attention. In this paper, by making some abstraction we study the key issues of the problem: (1) online content switching strategies, and (2) offline segmentation in preparation for adaptive streaming. Our work demonstrates how the problem depends fundamentally on user behavior, represented by user attention trajectories. Although we only relied on a typical real-world measured trajectory and two artificially generated benchmark trajectories, we point out how more knowledge of user attention can help optimize both the online and offline strategies.
\end{abstract}

\section{CCS CONCEPTS}

- Networks $\rightarrow$ Network performance evaluation; Network performance analysis; Application layer protocols;

\section{KEYWORDS}

Video streaming, bandwidth adaptation, virtual reality

\section{ACM Reference format:}

Hongwei Ma, Xiaoke Jiang, Rui Ma, Zhiyou Ma, Yizhen Cai, and Dah Ming Chiu. 2018. Smart Streaming of Panoramic Videos. In Proceedings of ACM SIGCOMM 2018 Morning Workshop on Virtual Reality and Augmented Reality Network, Budapest, Hungary, August 24, 2018 (VR/AR Network'18), 7 pages. https://doi.org/10.1145/3229625.3229628

\section{INTRODUCTION}

As more panoramic videos are made available online, the problem of how to do streaming of such videos has attracted lots of attention. The problem is both interesting and challenging - interesting because this problem brings up a new angle of adapting to user attention to the original streaming problem of adapting to only network bandwidth availability; challenging because the data rate requirement for these videos tend to be much higher and user attention is often less predictable than bandwidth availability.

There are lots of past studies and engineering solutions to video streaming, from fine-grained packet level protocols over UDP and

Permission to make digital or hard copies of all or part of this work for personal or classroom use is granted without fee provided that copies are not made or distributed for profit or commercial advantage and that copies bear this notice and the full citation on the first page. Copyrights for components of this work owned by others than the author(s) must be honored. Abstracting with credit is permitted. To copy otherwise, or republish, to post on servers or to redistribute to lists, requires prior specific permission and/or a fee. Request permissions from permissions@acm.org.

VR/AR Network'18, August 24, 2018, Budapest, Hungary

(C) 2018 Copyright held by the owner/author(s). Publication rights licensed to Association for Computing Machinery.

ACM ISBN 978-1-4503-5913-9/18/08 . \$15.00

https://doi.org/10.1145/3229625.3229628
RTP to coarse-grainded progressive downloading solution (see survey [2]). The most commonly accepted solution today is a chunk-bychunk based streaming protocol called DASH (Dynamic Adaptive Streaming over HTTP) [13]. The basic idea of DASH is to prepare multiple copies of each chunk of video, and as the available bandwidth between the user and video server changes, pick the copy of chunk to stream to optimize user's quality of experience (QoE). There exist many previous studies on adaptation strategies based on dynamic measurement and estimation of current and future available bandwidth $[5,9,12,16]$.

For panoramic videos streaming, there is a new interesting challenge. These videos typically require more bandwidth - due to the increase of content (going from viewing in one direction to viewing from all directions), as well as representation (going from 2D to $3 \mathrm{D}$ and often higher resolution). The obvious way to reduce the bandwidth requirement is to stream only that part of the video user is viewing in high definition. The feat, however, requires delicate video coding capability and a video streaming algorithm that adapts to user attention.

There are three video coding approaches to help adapt streamed videos to user attention:

- Foveated streaming: In this case, the video streamed to user is encoded in real time, in reaction to detected user attention. This approach does not seem to be very popular other than specialized situations, e.g. videos in games.

- Tile-based streaming: In this case, each video frame is partitioned (offline) into a set of tiles. Each tile has both highresolution and (possibly multiple) low resolution copies. For each streamed frame, the video server picks a high resolution tile based on currently detected user attention and use low(er) resolution copies for the rest of the tiles to stream to the video player. The player then composes the video frame based on received tiles. This is a popular approach currently studied by many $[3,4,6,7,10,14,15,17]$.

- Projection-based streaming: In this case, a number of copies of each video frame are prepared offline, each copy is encoded first by projecting the entire spherical view to a planar view, and then applying higher resolution encoding to some parts of the planar view to cover certain range of user attention. The streaming algorithm tries to switch to the best copy based on detected user attention. This approach is adopted by Facebook [8] and others, for example [11, 18].

These approaches require different levels of compute and storage support in the system. Foveated streaming requires fast compute support to dynamically encode and render the video at least at the server end; tile-based streaming requires more compute support at the player end to put the tiles together for rendering while the 
encoding can be done offline; for projection-based approach, the copies are all prepared offline and ready for rendering, but significantly more storage would be need in the servers and the CDN support this approach. But their goals are the same - reducing the required bandwidth while satisfying what user is viewing.

In this paper, our study is based on the Projection-based approach with a real world implementation by the co-authors from Kandao Technology. Also, in order to focus on the problem of adapting to user attention (rather than bandwidth variations at the same time), we assume the available bandwidth between server and player is given and is greater than the video playback rate. This assumption by no means trivializes the problem, but help zoom into the new problem of adapting to user attention.

The rest of the paper is organized as follows. In Section 2, we will give more background on how video coding is done for Projectionbased streaming, and define a QoE metric that is used to represent the overall goals of our problem. In Section 3, we define the copyswitching problem, which is caused by user attention changes over time and the fact there is always some delay in switching to a new copy. We use various user attention trajectories to study this problem, including a measured user attention trajectory as well as some artificially generated ones (e.g. random and scan) as benchmarks. We consider different switching algorithms and compare them to the offline optimal algorithm. In Section 4, we show the simulation results and discuss how different environmental factors can affect the streaming algorithm, such as the speed the user attention changes, the relative magnitude of playback rate compared to available bandwidth, and the number of copies available. In section 5, we briefly discuss the offline problem of segmentation, that is how many copies to prepare and what user attention areas they each cover. In Section 6, we conclude and discuss future directions.

\section{BACKGROUND AND ASSUMPTIONS}

\subsection{How copies are encoded}

In general, the panoramic videos are generated by special cameras covering a spherical view, like a globe view of the world. For human eyes, we first need to project the spherical view into a planar view, and there are many different planar representations. The simplest is the equi-rectangular projection; more sophisticated ones are cubemap, pyramid, and the further optimize offset cubemap and barrel projections explored by Facebook. For more explanations refer to $[8,18]$. In each of these representations, video encoding can allocate more pixels to that part of the planar view matching user's attention. For example, for the offset cubemap, it is the front rectangle. These straight-forward pixel allocation may not produce smoothly continuous pixel allocation across different parts of the planar view. For this reason, there is a equi-angular allocation for the cubemap representation which produces more smooth allocation, as shown in Fig 1. This is the approach taken by Kandao Technology, and is what we assume in defining the pixel allocation function.

\subsection{A measure of QoE}

In each encoded copy of the video, the allocation of pixels to different parts of the rendered video can be described by a pixel density function $p_{y}(x)$ where $y$ is the center of the copy. On the other hand, user's attention for the video can be captured by a focus of view

\section{Offcenter for pixel density reallocation}
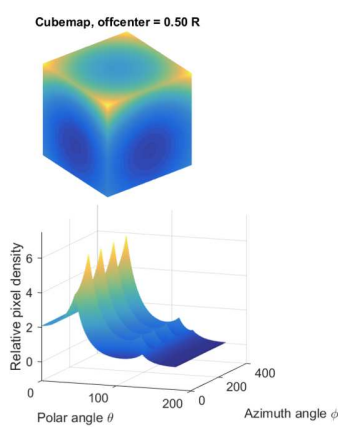
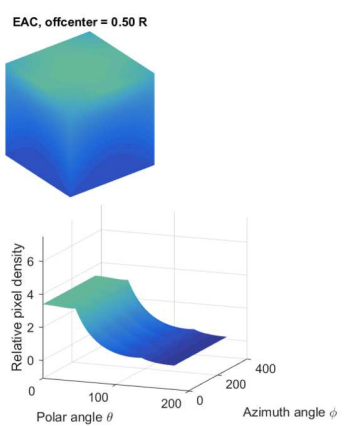

Figure 1: Some different projection methods and their resulting pixel density functions

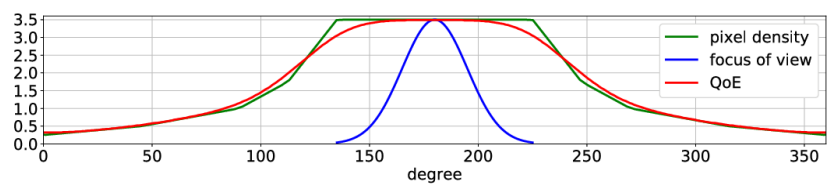

Figure 2: Illustration of how QoE $Q$ looks like based on a given pixel density function centered at 180 degrees

function $f_{z}(x)$ where $z$ is the center of the user's focus at time $t$. For simplicity, we can assume the video is only panoramic in the horizontal direction, so $x$ spans only the 360 degrees of radial dimen$\operatorname{sion}^{1}$. The user focus of view function $f_{z}(x)$ can be approximated by a Gaussian function, illustrated by the narrow curve in Fig 2. For the pixel density function $p_{y}(x)$, we use a square-edged function as illustrated in Fig 2, that is derived according to the projection and video encoding method for projection-based streaming described in the last subsection.

Based on $p_{y}(x)$ and $f_{z}(x)$, we can define a function for user QoE at time $t$ as

$$
Q(t)=\int_{0}^{2 \pi} f_{z(t)}(x) p_{y(t)}(x) d x
$$

where $y(t)$ references the copy used, and $z(t)$ is the center of focus of the user at time $t$. Clearly, this definition of user QoE is purely based on how much user attention is satisfied by the pixel density of the streamed copy of video. It is intended only for this study when there is never a problem with insufficient bandwidth ${ }^{2}$. In Fig 2, we also illustrates the QoE function as we move the user focus across different values of $x$, using the same copy at $y$ :

$$
Q_{y}(z)=\int_{0}^{2 \pi} f_{z}(x) p_{y}(x) d x
$$

In this figure, the value of $y$ is at 180 degrees, for both the $p_{y}(x)$ and $Q_{y}(x)$ curves.

\footnotetext{
${ }^{1}$ This assumption is considered a bit limitation of the current work by reviewers. We hope to address this in our future work.

${ }^{2}$ For video streaming adapting to network bandwidth, user QoE is often affected most severely by the shortage of available bandwidth, causing playback freezes or the use of low resolution copies.
} 
Another strong assumption in this study is that we only consider a horizontal pixel allocation function $p_{y}(x)$ whereas in real world pixel allocation also varies vertically, giving more resolution to the equator than the north and south poles. This is necessary to keep the discussion simple.

Establishing a quantitative QoE function $Q$ is important, as it gives us a basis for understanding and comparing different designs. We do not claim that $Q$ measures QoE exactly or highly accurately, but it is a reasonable and usable measure of QoE. Based on $Q$, we can derive insights from design analysis.

\subsection{Overall problem definition}

Broadly speaking, we have two problems: (a) Copy-switching algorithm design; (b) Segmentation design.

Copy-switching is an online algorithm. At any time $t$, the algorithm must determine whether the current copy is the best for the user compared to alternative copies of the video; if not, the process of switching to a new (better) copy is initiated. The challenge for the copy-switching strategy is that there is inevitably some delay from decision for a new copy and rendering of the new copy, and user attention can change quickly. A good switching algorithm needs to react smartly to changes in user reaction to achieve the best $Q$.

Note, in the switching problem, we assume the copies and the pixel density function for each are all given. Segmentation refers to the problem of deciding how many copies of the video to prepare (encode) and what each copy covers (i.e. each copies pixel density function). By definition, segmentation is a problem solved offline, even if the video itself is live, since the segmentation decisions need not necessarily depend on content. If the video is not live, hence the content is known at the segmentation time, that information can be helpful in predicting user attention. Similarly, if the video has been shown to other users and the user attention trajectories of previous viewing are available, that information can also be useful for both segmentation and switching strategies. Finally, as part of the offline problem, other parameters can be optimized. For example, the playback bit rate $R$ can be chosen based on the available bandwidth $B$, and expected speed user attention changes. A good investigation of the segmentation problem is found in [1].

In this paper, we will focus mostly on the simulation and analysis of the copy-switching strategy, with a brief discussion of the segmentation problem.

\section{COPY SWITCHING EVALUATION}

\subsection{User attention trajectory}

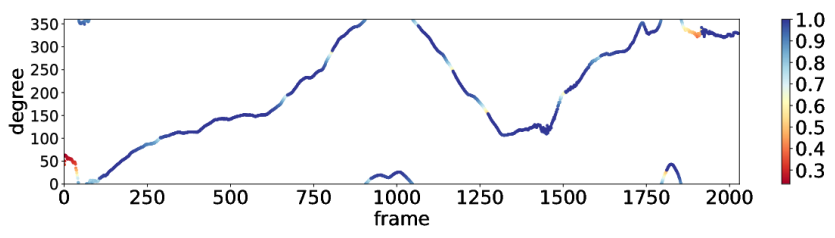

Figure 3: Illustration of a user flattened attention trajectory plotted against time
In order to discuss copy switching strategy, we need to introduce user attention trajectory, denoted by $u(t)$, that is simply the location of user attention at time $t$. Since we limit our discussion to only the horizontal dimension, $0 \leq u(t) \leq 2 \pi$ is a scalar quantify. Visually, $u(t)$ can be plotted against time, as in Fig 3, where the $y$-axis is the location from 0 to $2 \pi$ whereas the $x$-axis is time. What is shown in Fig 3 is a recorded real user attention trajectory. A small problem with this flattened representation of user attention is that it fails to show the fact that 0 and $2 \pi$ are actually the same point, and while $\delta$ and $2 \pi-\delta$ are two points very close to each other, they appear far apart in this representation. As a result, when user attention crosses the point $2 \pi$ (or 0 ), there appears to be a discontinuity in the trajectory. When we plot user trajectory, we can indicate the user QoE at the same time, using different colors, as illustrated by the color bar at the right hand side of the figure. To overcome the

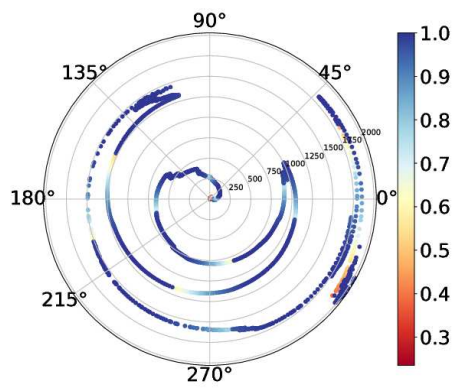

Figure 4: Illustration of the polar representation of user attention trajectory

problem with the flattened view, we can use a polar representation for the same user attention trajectory as shown in Fig 4. Here, each value of $u(t)$ is still represented by a dot, but the distance of the dot from the origin measures the time $t$ whereas the direction of $u(t)$ is the polar coordinate. Since each copy's coverage is approximated a sector of the circle, this representation helps visualizing which sector user attention tends to aggregate around. The color bar at the right hand side serves the same purpose as that in Fig 3 . This format also comes with its limitations. One problem is that speed of user attention change becomes distorted. Another problem is that it is harder to overlay other information in the polar plot, such as which copy is being used at a given time $t$. As a result, we choose to use mostly the first representation (as in Fig 3) when we discuss switching strategies. At the end, when we discuss segmentation, we will use the polar representation.

Besides real user attention trajectories, we will also use two artificially generated user attention trajectories for benchmark purposes. They are:

- scan trajectory: user's attention scans from position 0 towards position $2 \pi$ at a regular speed;

- random trajectory: user's attention is a random walk, this is generated in a similar way as the scan trajectory, except the direction of change can be either left or right.

For both of these benchmark trajectories, we can set the speed by adjusting the step size. In order to have useful comparison to the real user attention trajectories, we determine the average speed of 
the real trajectory, and use that for the benchmark trajectories. For the random trajectory, in order for it to have more coverage, we actually made it move "left" with probability 0.4 and move "right" with probability " 0.6 ", so it looks like a modified scan trajectory with some random zigzagging and a slower average rate in moving right. When discussing the performance of different streaming strategies for the real user trajectory, the performance on these artificial benchmarks help us get more insights into the challenges of the problem.

\subsection{Modeling copy switching with delay}

We assume the video streaming mechanism is based on a requestresponse protocol such as HTTP. There is a small delay between request and start of playback at the player, so some buffering is needed for continuous play. When switching from one copy to another copy, it is similar to starting a new video. It is necessary to avoid any freeze due to the initial delay. The basic technique is to overlay the unavoidable initial delay of the new stream with the continuation of the current video stream. At least two other factors affect the delay in switching from one copy to another. (a) an encoded video consists of a stream of GoPs (Group of Picture), and the starting point of playing a new copy must be at the beginning of a GoP; (b) a good amount of the current copy may already be buffered at the player side. From a bandwidth conservation point of view, switching to a new copy can be considered from both the QoE and bandwidth conservation point of view. In our simulation, however, we have not explicitly modeled the delay due to waiting for GoP boundaries, and have not considered bandwidth conservation. Instead, we have simply used a suitable value for the minimum initial delay from request to playback.

Since there is a delay during the process of copy switching, how should the switching algorithm evaluate the value of $Q$ for different copies to minimize the likelihood of misjudging the merit of each copy by the time switching to the new copy is realized? Since the switching strategy is an online algorithm, the knowledge of user attention available to the algorithm is only the current position $u(t)$. Clearly, the correct copy to switch to depends on the knowledge of $u\left(t^{\prime}\right)$ where $t^{\prime}$ is the time switching happens, but value of $u\left(t^{\prime}\right)$ is unknown to the switching algorithm.

For our analysis, we evaluate the following switching strategies:

(1) baseline strategy (B): In this case, we assume $u\left(t^{\prime}\right)=u(t)$ for $t^{\prime}>t$ when calculating $Q$ for different copies;

(2) simple Markov estimator strategy (M): In this case, we make a directional adjustment which is based on a simple Markov model. If the user attention has not changed in the immediate past, we assume it will stay the same with $50 \%$ chance and change to each direction with $25 \%$ chance each; if the user attention moved from left to right in the immediate past, then we assume it will continue to move right with $50 \%$ chance, and stay stationary or move left with $25 \%$ chance each; and likewise for the case of moving left in the immediate past.

(3) distance estimator strategy (D): In this case, the position of $u\left(t^{\prime}\right)$ is estimated for some expected $t^{\prime}$ (when switching is expected to happen). The estimation is based on the speed of attention change in the recent past. Note, when there is a turn-around in user attention movement, the distance estimator takes note of the new direction and estimates the distance based on the speed of user attention moving in the new direction.

(4) offline optimal strategy (O): In this case, we assume the best copy is used at each $t$.

We study these strategies based on simulation, using different types of user attention trajectories mentioned above.

\section{SIMULATION RESULTS}

For all the simulation experiments in this section (unless indicated otherwise), we assume there are four copies of the video, each covering a different 90 degree quadrant, and the center of these four copies are respectively 45, 135, 225, 315 degrees. At the beginning of viewing, a random copy is used (this is only the case for these simulation experiments; in real implementation, it is easy to be smarter). The available bandwidth is $B=33$ and playback rate is $R=30$ (unit not given as only relative magnitude matter).

\subsection{Real versus benchmark}

We first show how the baseline switching algorithm (the one that assumes the observed current position of use attention will remain unchanged till a new copy is streamed) works for the three user attention trajectories: (a) measured trajectory, (b) scan trajectory and (c) random trajectory. The three different trajectories and the copies used by the base algorithm are shown in Figure 5(a), 6(a) and 7(a). Figure 5(b), 6(b) and 7(b) show the variation of the instantaneous value of $Q(t)$ for each case. In each figure, the first plot

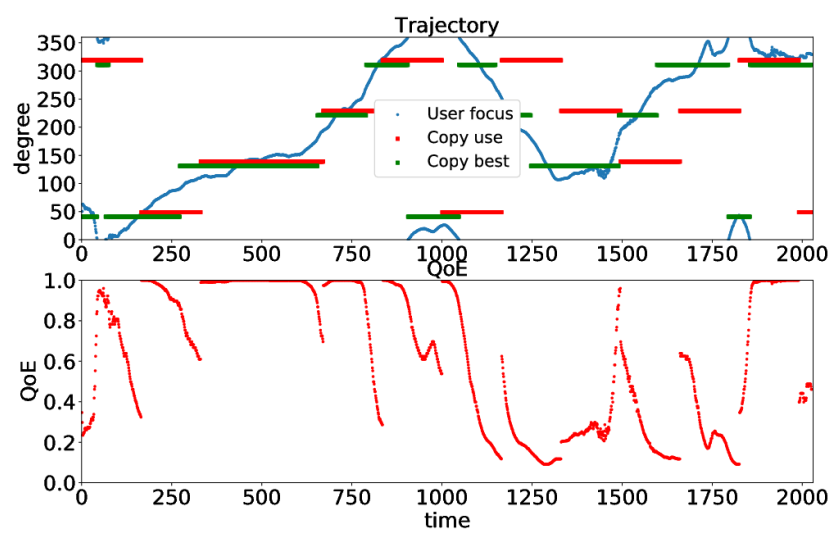

Figure 5: Performance of baseline strategy on real user trajectory, average $Q$ is 0.63

shows the user attention trajectory with the use of different copies indicated by lines spanning the duration of use, and $y$-axis value (the center of a copy) identifying the copy in use. Actually, each copy is represented by two lines one on top of the other - they represent the timing of a copy selected, and the timing of that copy actually being streamed. The latter is always slightly delayed for reasons explained in Section 3.2. The second plot for each above figure shows $Q(t)$ over time. 


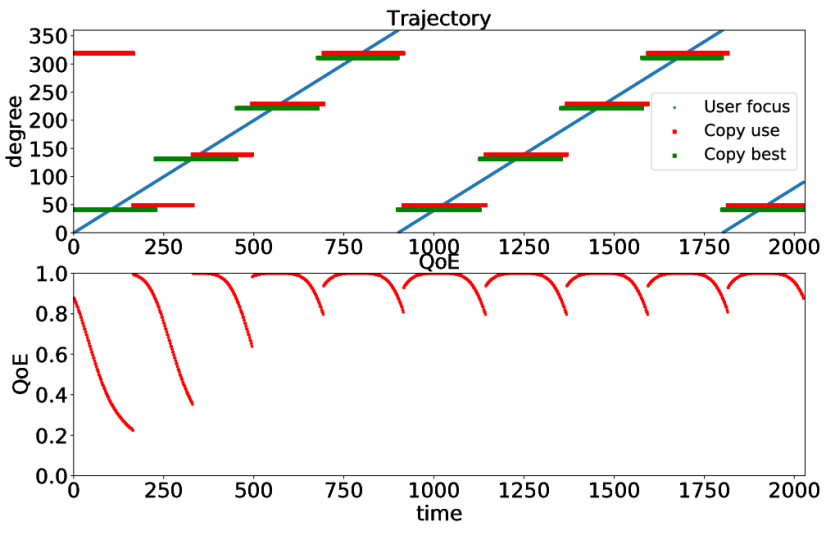

Figure 6: Performance of baseline strategy on scan trajectory, average $Q$ is 0.91

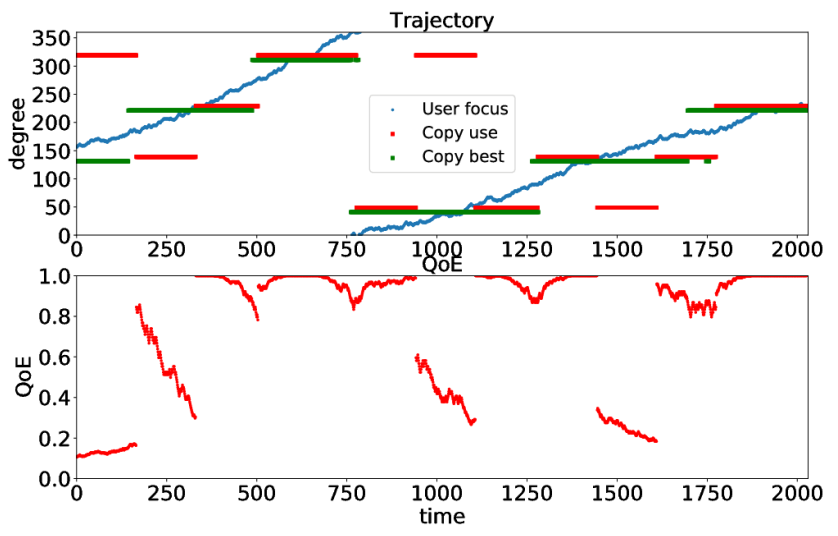

Figure 7: Performance of baseline strategy on random trajectory, average $Q$ is 0.76

As expected, since the scan trajectory is most predictable, its performance is also most regular. For the random trajectory, the performance varies more, and occasionally a wrong copy is streamed and it takes some time to correct. At this particular $B$ and $R$ ratio (33 and 30), the real user trajectory performed quite poorly, even worse than the random case (though at higher ratios of $B$ to $R$, we found the random trajectory is usually the most challenging). The lost of QoE occurs mostly during switching. This is due to the discrepancy between best copy and the delay in successfully switching to it. To improve QoE, obviously we need to either speed up switching (hard to do) or do better prediction.

\subsection{The effect of speeding and bandwidth}

Before we discuss how to do better prediction of user attention, we show the effect of some system parameters, namely (a) the relative value of playback rate $R$ to available bandwidth $B$, and (b) the speed of change of user attention, to switching performance. We show both in Fig 8. For this experiment, we used the scan trajectory and the baseline switching strategy. As expected, the switching performance here is quite good at low speed, since hardly any switching

\begin{tabular}{|l|c|c|c|}
\hline & real & scan & random \\
\hline baseline & 0.63 & 0.91 & 0.76 \\
\hline Markov & 0.68 & 0.89 & 0.83 \\
\hline Distance & 0.89 & 0.92 & 0.86 \\
\hline Offline & 0.980 & 0.975 & 0.971 \\
\hline
\end{tabular}

Table 1: Comparison of user attention prediction algorithms using different user attention trajectories

needs to be done. As we increase the speed of user attention change, the performance in terms of average $Q$ gets worse quickly due to more and more switching of copies, each time incurring some loss of $Q$. What is unexpected is that when speed becomes higher than some threshold, the performance recovers to a level better than the worst performance level. By looking closely at the copies used for each part of user trajectory, we find that the faster attention changes, the worse is the improvement of the new copy; so when the speed moving in the same direction (scan trajectory) is fast enough, it eventually becomes better to be limited in how often you switch.

It is interesting the effect of adjusting $B / R$ has a very similar effect. In most experiments, we set $B=33$ and $R=30$. As we vary $B / R$ from 1 to 1.3 , with speed equal to 1 , then we stop gaining any performance beyond $B / R=1.2$ (for different switching algorithms, we will level off at different heights). But as $B / R$ is reduced, there is less time to buffer content hence limiting the ability to react to changes in user attention, hence worse QoE. But again, when the ability to react becomes slow beyond a certain threshold, the delay in switching to a new copy is so slow that it becomes better to switch less.

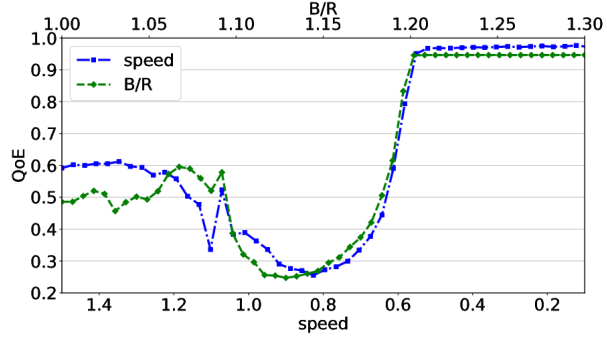

Figure 8: Effect of changing $R$ (relative to $B$ ) and speed of user attention change. When $B / R$ is varied, speed is 1 ; when speed is varied, $B=33$ and $R=30$

\subsection{Comparing different switching strategies}

Now we show how prediction of user attention can improve QoE when compared to the baseline algorithm. We show two prediction algorithms for user attention as described in the last section, referred to as Simple Markov and Distance-prediction (or Markov and Distance for short). As a benchmark, we also include the offline optimal solution (assuming perfect knowledge and no delay in switching). The results are summarized in Table 1.

Recall that the simple Markov model is just a very rough, incremental correction for the baseline algorithm, hence it improves the 
baseline only slightly, except for the scan trajectory the Markov strategy actually gets slightly worse. This is understandable since it makes a mistake a fraction of the time (by moving left). The Distance algorithm improves QoE significantly more, and improves in each case. The offline optimal QoE, as expected, gives the upper bound to all these strategies. Note, the offline optimal is not $100 \%$ either, since we are only using four copies to cover the 360 degree video. Actually, this indicates that a segmentation into four copies can potentially do quite well, if we can further optimize prediction performance.

\section{SEGMENTATION}

\subsection{The effect of increasing copies}

As we mentioned in Section 2, part of the overall streaming problem is to decide how many copies to use and what each copy covers we called this segmentation. For the switching problem, the copies are given. Thus far, we have assumed the simplest case of using four copies, each covering a different $90 \%$ quadrant. Now, we are going to experiment (by simulation) using more copies and see if QoE will improve. When increasing number of copies, we assume the copies are used to cover evenly divided intervals of the $360 \%$ spectrum. In practice, if there is knowledge of where user attention tends to focus on, e.g. if we know the heat map, then it is possible to be smarter in using more copies, but that will also be our future direction.

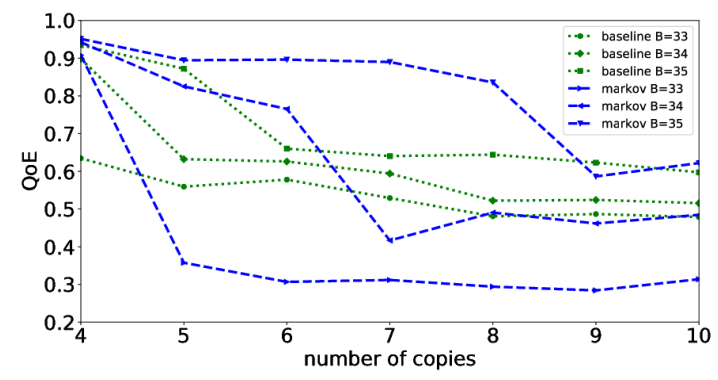

Figure 9: Effect of increasing the number of copies, assuming regular coverage by each copy.

In Fig 9, we show the performance of switching when the number of copies is increased from 4 to 10 . We would have expected there were some improvements in performance, even only moderately. But to our surprise, the performance actually worsened uniformly. By always making the copies center at regular intervals of the 360 degree spectrum, more copies tend to lead to more switching, and we know that the loss of QoE occurs mostly during switching. This is a counter-intuitive result. The lesson is, when we use more copies it is important to decide carefully what each copy covers based on user attention analysis. Therefore, more copies means more challenge in designing good switching algorithms.

\subsection{Shifting copy coverage to improve QoE}

While the entire treatment of the segmentation problem is complicated, one idea is simple to try out. Assume we still just use four copies to cover the 360 degree video, we can choose the four quadrants they cover. In other words, they don't have to be centered at
$45,135,225,315$ degrees. Instead, we can shift their center (together) so that a best alignment that minimizes number of switching. This can be easily done offline if user attention trajectory is known. We plot the result of two specific orientations (note, once you decide the coverage of one copy, the other three are also decided since they together must cover the 360 degrees), one is at 34 degrees and one is at 68 degrees. We use the polar representation together with $Q$ values to show the results. In this representation, we can see clearly

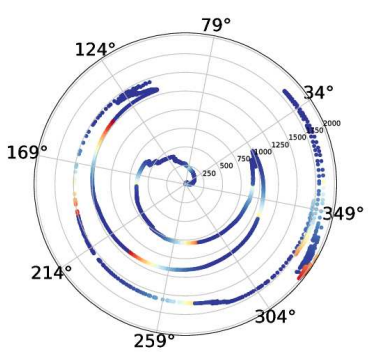

Figure 10: Copies shifted to start at 34 degrees

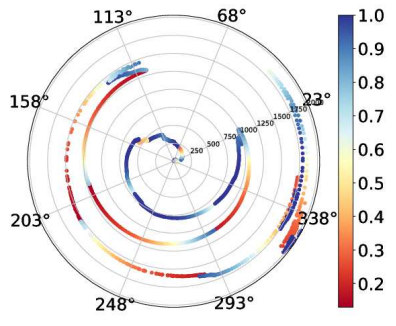

Figure 11: Copies shifted to start at 68 degrees that aligning the copies to start at 34 degrees works much better than 68 degrees, and how the trajectory is covered by the different copies all at once. Of course, this is based on offline calculation, i.e. based on information that may not be available, at least in the live video case. But this example and analysis shows that there is much to explore in how to align the copies to user attention, gathered either from lots of past trajectory measurement, or estimated from content analysis. This direction is also for our future works.

\section{CONCLUSION}

In this paper, we studied the problem of streaming panoramic video (based on the projection-based approach) by making some assumptions and problem abstraction. This allows us to highlight how both online switching and offline segmentation strategies critically depend on user attention behavior. Through collaboration with Kandao Technology, much of the work is based on experiences in their high performance implementation of such a streaming system. Due to space limitation, we plan to share that aspect through another publication or technical report.

Based on this foundation, there are several directions for future research. The design of the QoE function can be improved based on subjective evaluation. For example, the QoE may also depend on the smoothness of the video, which is not considered in our QoE function. It is important to extend the current horizontal only model to a two dimensional model. Another direction is to revisit the learning and control strategies in adaptive streaming. We have collected a large sample of user attention trajectories by posting some of our panoramic videos online. we plan to follow the approach in [9], which tried to improve DASH performance by learning available bandwidth change patterns over time from a large sample of measurement data. Finally, the segmentation problem can also take advantage of the user trajectory measurement data that we have collected. 


\section{REFERENCES}

[1] X Corbillon, A Devlic, G Simon, and J Chakareski. 2017. Optimal Set of 360-Degree Videos for Viewport-Adaptive Streaming. In ACM Multimedia.

[2] D Wu et al. 2001. Streaming video over the Internet: approaches and directions. 11 (2001), 282-300. Issue 3

[3] K Misra et al. 2013. An overview of tiles in HEVC. IEEE fournal of selected topics in signal processing 7, 6 (2013), 969-977.

[4] L Xie et al. 2017. 360ProbDASH: Improving QoE of 360 Video Streaming Using Tile-based HTTP Adaptive Streaming. In ACM Multimedia.

[5] TY Huang et al. 2014. A Buffer-based Approach to Rate Adaptation: Evidence from a Large Video Streaming Service. In ACM Sigcomm.

[6] J Le Feuvre and C Concolato. 2016. Tiled-based adaptive streaming using mpegdash. In ACM MMSys.

[7] A Ghosh, V Aggarwal, and F Qian. 2017. A Rate Adaptation Algorithm for Tile-based 360-degree Video Streaming. arXiv preprint arXiv:1704.08215 (2017).

[8] E Kuzyakov, S Chen, and R Peng. 2016. Enhancing highresolution 360 streaming with view prediction. (2016) https://code.facebook.com/posts/1126354007399553/next-generation-videoencoding-techniques-for-360-video-and-vr/.

[9] H Mao, R Netravali, and M Alizadeh. 2017. Neural Adaptive Video Streaming with Pensieve. In ACM Sigcomm.

[10] S Petrangeli, V Swaminathan, M Hosseini, and F De Turck. 2017. An HTTP/2based adaptive streaming framework for $360 \mathrm{i} f_{i}$ virtual reality videos. In $A C M$ Multimedia.

[11] Pixvana. 2017. An Intro to FOVAS: Field of View Adaptive Streaming for Virtual Reality. (2017). http://blog.pixvana.com/intro-to-field-of-view-adaptivestreaming-for-vr/.

[12] K Spiteri, R Urgaonkar, and RK Sitaraman. 2016. Near-Optimal Bitrate Adaptation for Online Videos. In IEEE Infocom.

[13] T Stockhammer. 2011. Dynamic adaptive streaming over HTTP: standards and design principles. In ACM MMSys. 133-144.

[14] A Taghavinasrabadi, A Mahzari, J Beshay, and R Prakash. 2017. Adaptive 360degree video streaming using layered video coding. In Virtual Reality.

[15] M Xiao, C Zhou, Y Liu, and S Chen. 2017. OpTile: Toward Optimal Tiling in 360-degree Video Streaming. In ACM Multimedia.

[16] X Yin, A Jindal, V Sekar, and B Sinopoli. 2015. A Control-Theoretic Approach for Dynamic Adaptive Video Streaming over HTTP. In ACM Sigcomm.

[17] A Zare, A Aminlou, M Hannuksela, and M Gabbouj. 2016. HEVC-compliant tile-based streaming of panoramic video for virtual reality applications. In $A C M$ Multimedia.

[18] C Zhou, Z Li, and Y Liu. 2017. A Measurement Study of Oculus 360 Degree Video Streaming. In ACM MMSys. 